\title{
Erratum to: Twins in spirit: DOTATATE and high-affinity DOTATATE
}

\author{
Claudia Brogsitter • Margret Schottelius • Klaus Zöphel • \\ Jörg Kotzerke • Hans-Jürgen Wester
}

Published online: 21 September 2013

(C) Springer-Verlag Berlin Heidelberg 2013

Erratum to: Eur J Nucl Med Mol Imaging

DOI 10.1007/s00259-013-2497-5

The legend to the figure should read:

A- MIP ${ }^{68} \mathrm{Ga}$-DOTATATE

B- MIP ${ }^{68} \mathrm{Ga}$-HA-DOTATATE

The online version of the original article can be found at http://dx.doi.org/ 10.1007/s00259-013-2497-5.

C. Brogsitter $\cdot$ K. Zöphel $\cdot$ J. Kotzerke $(\bowtie)$

Klinik und Poliklinik für Nuklearmedizin, Universitätsklinikum Carl

Gustav Carus, Technischen Universität Dresden, Dresden, Germany

e-mail: joerg.kotzerke@mailbox.tu-dresden.de

M. Schottelius $\cdot$ H.-J. Wester

Pharmaceutical Radiochemistry and Department of Nuclear

Medicine, Technische Universität München, Munich, Germany 